\title{
Diabetes and its Resulting Complications: Mathematical Modeling via Fractional Calculus
}

\section{Srivastava $\mathrm{HM}^{*}$}

Department of Mathematics and Statistics, University of Victoria, Canada

Mini Review

Volume 4 Issue 2

Received Date: September 14, 2020

Published Date: October 20, 2020

DOI: $10.23880 /$ phoa- 16000163 University of Victoria, Victoria, British Columbia V8W 3P4, Canada; Email: harimsri@math. uvic.ca

\section{Abstract}

Our main objective in this editorial-cum-mini review article is to briefly outline several recent investigations in which mathematical analytic studies were successfully made of the model of diabetes and its resulting complications by means of the widely- and extensively-applied concept of fractional-order calculus, not only in the medical and biological sciences, but also in the physical, chemical, statistical and engineering sciences.

Keywords: Fractional Calculus; Diabetes Mellitus; Glucose

Abbreviations: DM: Diabetes Mellitus; GDP: Global gross Domestic Product; GTT: Glucose Tolerance Test; IDF: International Diabetes Federation; HDM: Homotopy Decomposition Method;

\section{Introduction}

This Editorial-cum-Mini Review is motivated essentially by an Opinion expressed recently in this journal by Fitria Najmiatul [1], which advises that "Pregnant women with increasing blood glucose levels are identified as being more exposed to the severe effects of COVID-19 and advised to follow social distancing stringently."

Diabetes is a worldwide problem which effects 1 out of every 11 persons now- a-days. The IDF Diabetes Atlas (Eighth Edn, 2017) states that approximately 415 million people in the world are living with the disease and that this number will rise to 629 million by the year 2045. It is a very serious problem of the world. A major part of the world population is affected by this disease and its resulting complications. Diabetes mellitus (DM), which is also known as a Silent Killer in medical science, is a metabolic disorder which occurs when the sugar level in blood is not appropriate. The glucose in human blood is the main source of energy and comes from the food insulin, a hormone made by the pancreas, which helps glucose from food to get into our cells and to use it for energy. Insulin also controls and adjusts the quantity of sugar in human body [2]. When a human body is unable to make the desired amount of insulin or is not able to use it well, then the glucose doesn't reach the cells and stays in the blood. Frequent urination (polyurea), feeling more thirsty and hungry (polydipsia and polyphagia) are the symptoms which the patients having high blood sugar typically experience. Diabetes is known as a Raj Rog (meaning "kingship disease" or "royalty dis- ease") in ancient India, that is, a disease which affects those people having lots of wealth and lives a comfortable prosperous life by using servants for doing their works and chores. But, in recent years, it has become a problem which affects the whole society. In ancient times, this problem was seen in the age group above 70 years, but (in today's era) it is a problem of all age groups. And, in very short time period, it has become a problem of the whole world. The issue is very serious and researchers must contribute their best to control this problem.

Generally, Diabetes is considered to be of the following two types.

- Type I Diabetes: Type I diabetes requires a daily dose of insulin taken by the patient to regulate the amount of 
glucose in blood. Non-access of insulin leads to certain complications or danger to the patient's life. The cause of the Type I diabetes is not known as yet and is currently not preventable. Symptoms include excessive and frequent urination and thirst, constant hunger, weight loss, vision change and fatigue.

- Type II Diabetes: In this type of diabetes, we consider those patients who do not depend on insulin. For Type II diabetes, the doctor recommends the patient to follow a particular diet chart and exercise. By means of this restriction, one can control one's Type II diabetes. Yoga also plays a very efficient role in order to control the Type II diabetes. Symptoms of the Type II diabetes may be similar to those of the Type I diabetes, but are often less marked or absent. Diabetes is steadily increasing everywhere not only in adults, but also in children, most markedly in the world's middle-income countries [3]. There is a prediction on the basis of the increasing number of diabetes patients that, in the world, the diabetes cases can jump to 55 percent in 2035.

Diabetes mellitus (DM), which is also known as a Silent Killer in medical science, is a metabolic disorder which occurs when the sugar level in blood is not appropriate. The glucose in human blood is the main source of energy and comes from the food insulin, a hormone made by the pancreas, which helps glucose from food to get into our cells and to use it for energy. Insulin also controls and adjusts the quantity of sugar in human body [4]. When a human body is unable to make the desired amount of insulin or is not able to use it well, then the glucose doesn't reach the cells and stays in the blood. Frequent urination (polyurea), feeling more thirsty and hungry (polydipsia and polyphagia) are the symptoms which the patients having high blood sugar typically experience.

Diabetes is known as a Raj Rog in ancient India, that is, a disease which affects those people having lots of wealth and lives a comfortable prosperous life by using servants for doing their works and chores. But, in recent years, it has become a problem which affects the whole society. In ancient times, this problem was seen in the age group above 70 years, but (in today's era) it is a problem of all age groups. And, in very short time period, it has become a problem of the whole world. The issue is very serious and researchers must contribute their best to control this problem.

The International Diabetes Federation (IDF) had recently given the information to the effect that the top 5 countries in the world have highest numbers of diabetes patients (Table 1).

\begin{tabular}{|c|c|c|}
\hline Name of the country & Total population & People affected by diabetes \\
\hline People's Republic of China & $1,41,13,16,334$ & 109.6 million \\
\hline India & $1,33,91,80,127$ & 69.2 million \\
\hline U.S.A. & $32,44,59,463$ & 30.3 million \\
\hline Brazil & $20,92,88,278$ & 14.2 million \\
\hline Russian Federation & $14,39,81,725$ & 12.5 million \\
\hline
\end{tabular}

Table 1: Top 5 Countries by the Number of Diabetes Patients.

There are some other countries also that have the highest proportion of people living with diabetes such as Tokelau (29.7\%), Mauritius (24.3\%), Nauru (23.8\%), Cook Islands (21.1\%), and Marshall Islands (21.1\%) [6].

Complications: All types of Diabetes can lead to various diseases and health problems such as (for example) heart attack, kidney failure, lower limb amputation, blindness and nerve damage. In fact, diabetes increases the overall risk of dying prematurely. For a pregnant woman, uncontrolled diabetes increases the risk of fetal death and other complications. Economic Impact: According to a new research conducted at the University of Gottingen and at the Harvard T. H. Chan School of Public Health, which was published online on April 26, 2017 in The Lancet Diabetes \& Endocrinology, the world faces a notable economic burden from diabetes, that is, about $1.8 \%$ of global gross domestic product (GDP). The study also found that, not only the direct costs of the disease such as (for example) expenditures for insulin, testing strips and treatment, but also the indirect costs representing production shortfalls due to illness and premature death accounts for nearly $35 \%$ of the total economic burden [7-16].

Prevention of Diabetes: Type II diabetes can be prevented by losing weight and following a regular healthy diet and exercise chart, but Type I diabetes cannot be prevented with our current knowledge till today. Resistance training (twice a week) and regular aerobic exercise maintain blood sugar level and are very effective in controlling diabetes. WHO and FAO provide guidance for diet to prevent Type II Diabetes [11]. WHO also recommends different physical activities for different age groups [17], because physical activity and diet are significantly more effective than medication? Diabetes 
is diagnosed by the Glucose Tolerance Test (GTT) in which glucose level is measured by blood sample, while the patient is in a fasting state. Diagnosis of diabetes of a patient in nonfasting state can be done by the measurement of glycated hemoglobin (HbA1c) which reflects the average blood glucose concentration. However, this test is more expensive than blood glucose measurement.

Here, in this Editorial-cum-Mini Review, we choose to briefly review and outline a recent investigation by Srivastava, et al. [18] in which a mathematical study was made of model of diabetes and its resulting complications by means of the widely- and extensively-used concept of fractional calculus. The parameters of the mathematical model define the population of diabetic patients and those who are diabetic with complications at a given time t. We have also discussed the existence, uniqueness and stability of the fractional-order model which we consider here. We make use of the homotopy decomposition method (HDM) in order to solve the problem.

\section{An Introductory Overview of Fractional Calculus}

In the study and analysis of various real-world problems, the subject of fractional calculus has played a significant role in their mathematical modeling. It is capable of modeling and analyzing linear as well as complex phenomena of engineering, biological and applied sciences. In modern times, fractional calculus plays very essential role in such fields as (for example) biology, geophysics, biomedical engineering, fluid dynamics, electricity, and mechanics. It has thus become a very efficient tool to describe significant models of the natural phenomena. Many types of problems can be defined by the help of fractional-order integration and fractional-order differentiation. These include, but are not limited to, stochastic processes, blood glucose insulin model, Brownian motion, fractional oxygen diffusion problem, control theory, viscoelasticity, fractals and nonlocal phenomena, time-fractional telegraph equation, spacetime fractional Fokker-Plank equation, fractional LC-circuit models, fractional Klein-Gordon equations, heat conduction, image and signal processing, control theory, controllability of fractional delay dynamical systems [19-30], in each of which one can find a great deal of literature on fractional calculus and its wide-spread applications.

\section{Fractional-Order Modeling of Diabetes: A Brief Account}

Basing their investigation essentially upon the description of the mathematical model of diabetes and its resulting complications, which was considered in the year 2004 by Boutayeb, et al. [31], Srivastava, et al. [18] considered a model, in which $C(t)$ and $B(t)$ represent patients with and without complications, respectively, at time $t$, and assumed that the total number of diabetic patients at a time $t$ is $E(t)$, so that

$$
E(t)=B(t)+C(t)
$$

In terms of the celebrated Riemann-Liouville fractional derivative operators $D_{t}^{\alpha}$ and $D_{t}^{\beta}$ of fractional orders $\alpha$ and $\beta$, respectively, their fractional-order mathematical model is governed by the following system of fractional differential equations [18].

$$
\begin{gathered}
D_{t}^{\alpha}[B(t)]=A(t)-(\delta+\varepsilon) B(t)+\lambda C(t) \\
(t>0 ; 0<\alpha \leqq 1) \\
D_{t}^{\beta}[C(t)]=A(t)-(\lambda+\mu+v+\varepsilon) C(t)+\sigma B(t) \\
(t>0 ; 0<\beta \leqq 1)
\end{gathered}
$$

Together with

$$
C(0)=C_{0} \quad \text { and } \quad E(0)=E_{0}
$$

Equivalently, we have

$$
\begin{gathered}
\mathrm{D}_{t}^{\alpha}[C(t)]=-(\delta+\varphi) C(t)+\delta E(t) \\
(t>0 ; 0<\alpha \leqq 1)
\end{gathered}
$$

And

$$
\begin{gathered}
D_{t}^{\beta}[E(t)]=A(t)-(\mu+v) C(t)-\varepsilon E(t) \quad(2.6) \\
(t>0 ; 0<\beta \leqq 1)
\end{gathered}
$$

Respectively, together with

$C(0)=C_{0} \quad$ and $\quad E(0)=E_{0}$

Just as in Eq. (2.4).

By considering the solution of the above-described problem for different values of the parameters involved therein, the importance of fractional-order model, and by means of the illustrative graphical representations of the obtained results, it was observed that the population $\mathrm{E}(\mathrm{t})$ of those patients who have diabetes at time $t$ for $\alpha=\beta=1$ decreases speedily in comparison to the case for fractional values of the orders $\alpha$ and $\beta$. Nevertheless, it is clear from researches and the WHO report that, in the present scenario, it is not the case. Hence, from the results derived and conclusions made in the paper [18], it is made clear that the fractional-order model is better to describe the problem of diabetes and its resulting complications. 


\section{Conflict of Interest}

The author declares no conflict of interest.

\section{References}

1. Najmiatul F (2020) Gestational Diabetes Mellitus and Pandemic Covid-19: Where do we Stand?. Public H Open Acc 4(2): 000155.

2. WHO (1999) Definition, Diagnosis and Classification of Diabetes Mellitus and Its Complications: Report of a WHO Consultation. Part 1: Diagnosis and Classification of Diabetes Mellitus.

3. WHO (2016) Report Commission, Global Report on Diabetes.

4. Whiting D, Unwin N, Roglic G (2010) Diabetes: Equity and social determinants, In Equity, Social Determinants and Public Health Programmes. Blas E, et al. (Eds.), World Health Organization, Geneva, pp: 77-94.

5. NCD Risk Factor Collaboration (NCD-RisC) (2016) Worldwide trends in diabetes since 1980: A pooled analysis of 751 population-based studies with 4.4 million participants. Lancet 387(10027): 1513-1530.

6. Diamond Project Group (2006) Incidence and trends of childhood type 1 diabetes worldwide 1990-1999. Diabetes Medicine 23(8): 857-866.

7. WHO (2015) Guideline, Sugars Intake in Adults and Children.

8. Morenga LT, Mallard SJM (2012) Dietary sugars and body weight: Systematic review and Meta analyses of randomized controlled trials and cohort studies, BMJ 346: 1-25.

9. WHO (2014) Global Status Report on Noncommunicable Diseases.

10. WHO (2013) Global Action Plan for the Prevention and Control of Noncommunicable Diseases 2013-2020.

11. WHO (2003) Diet, Nutrition and the Prevention of Chronic Diseases. Report of a Joint WHO/FAO Expert Consultation, pp: 1-160.

12. Johnsson W, Haglund B, Ahlsson F, Gustafsson J (2015) A high birth weight is associated with increased risk of type 2 diabetes and obesity. Pediatr Obes 10(2): 77-83.

13. Whincup PH, Kaye SJ, Owen CG, Rachel H, Derek GC, et al. (2008) Birth weight and risk of type 2 diabetes: A systematic review. JAMA 300(24): 2886-2897.

14. Harder T, Rodekamp E, Schellong K, Dudenhausen JW, Plagemann A (2007) Birth weight and subsequent risk of type 2 diabetes: A meta-analysis. Am J Epidemiol 165(8): 849-857.

15. Willi C, Bodenmann P, Ghali WA, Faris PD, Cornuz J (2007) Active smoking and the risk of type 2 diabetes: A systematic review and meta-analysis. JAMA 298(22): 2654-2664.

16. Luo J, Rossouw J, Tong E, Giovino GA, Lee CC, et al. (2013) Smoking and diabetes: Does the increased risk ever go away? Am J Epidemiol 178(6): 937-945.

17. WHO (2010) Global Recommendations on Physical Activity for Health, pp: 1-60

18. Srivastava HM, Dubey RS, Jain M (2019) A study of the fractional-order mathematical model of diabetes and its resulting complications. Mathematical Methods in the Applied Sciences 42(13): 4570-4583.

19. Podlubny (1999) Fractional Differential Equations: An Introduction to Fractional Derivatives, Fractional Differential Equations, to Methods of Their Solution and Some of Their Applications, Academic Press 198: 1-340.

20. Miller KS, Ross B (1993) An Introduction to Fractional Calculus and Fractional Differential Equations, A WileyInterscience Publication. John Wiley and Sons.

21. Kilbas AA, Srivastava HM, Trujillo JJ (2006) Theory and Applications of Fractional Differential Equations, NorthHolland Mathematical Studies 204: 1-523.

22. Srivastava HM (2020) Fractional-order derivatives and integrals: Introductory overview and recent developments. Kyungpook Mathematical Journal 60(1): 73-116.

23. Srivastava HM, Günerhan H (2019) Analytical and approximate solutions of fractional-order susceptibleinfected-recovered epidemic model of childhood disease. Mathematical Methods in the Applied Sciences 42(3): 935-941.

24. Alkahtani BS, Alkahtani JO, Dubey RS, Goswami P (2017) The solution of modified fractional Bergman's minimal blood glucose-insulin model. Entropy 19(5): 1-11.

25. Atangana A, Alabaraoye E (2013) Solving system of fractional partial differential equations arising in the model of HIV infection of CD4+ cells and attractor one-dimensional Keller-Segel equations. Advances in Difference Equations, pp: 1-14. 


\section{Public Health Open Access}

26. Dubey RS, Belgacem FBM, Goswami P (2016) Homotopy perturbation approximate solutions for Bergman's minimal blood glucose-insulin model. Fractal Geometry and Nonlinear Anal in Med and Biol 2(3): 1-6.

27. Srivastava HM, Saad KM, Khader MM (2020) An efficient spectral collocation method for the dynamic simulation of the fractional epidemiological model of the Ebola virus. Chaos Solitons Fractals 140(110174): 1-7.

28. Singh H, Srivastava HM (2020) Numerical simulation for fractional-order Bloch equation arising in nuclear magnetic resonance by using the Jacobi polynomials. Applied Sciences 10(8): 1-18.
29. Srivastava HM, Saad KM, Gómez-Aguilar JF, Almadiy AA (2020) Some New Mathematical Models of the Fractional-Order System of Human Immune against IAV Infection. Mathematical Biosciences and Engineering 17(5): 4942-4969.

30. Srivastava HM (2020) Operators of Basic (or $q$-) Calculus and Fractional $q$-Calculus and Their Applications in Geometric Function Theory of Complex Analysis. Iranian Journal of Science and Technology Transaction A-science 44: 327-344.

31. Boutayeb A, Twizell EH, Achouayb K, Chetouani A (2004) A mathematical model for the burden of diabetes and its complications. Biomed Eng Online 3(1): 1-8. 